\title{
SHORT REPORT \\ Chlamydia gallinacea: a widespread emerging Chlamydia agent with zoonotic potential in backyard poultry
}

\author{
L. LI ${ }^{1}$, M. LUTHER ${ }^{1}$, K. MACKLIN ${ }^{2}$, D. PUGH ${ }^{1}$, J. LI $^{3}$, J. ZHANG $^{3}$, \\ J. ROBERTS ${ }^{1}$, B. KALTENBOECK ${ }^{4}$ AND C. WANG ${ }^{4 *}$ \\ ${ }^{1}$ Thompson Bishop Sparks State Diagnostic Laboratory, Auburn, AL, USA \\ ${ }^{2}$ Department of Poultry Science, College of Agriculture, Auburn University, AL, USA \\ ${ }^{3}$ Department of Basic Sciences, College of Veterinary Medicine, Yangzhou University, Yangzhou, China \\ ${ }^{4}$ Department of Pathobiology, College of Veterinary Medicine, Auburn University, AL, USA
}

Received 8 May 2017; Final revision 24 June 2017; Accepted 9 July 2017;

first published online 3 August 2017

\section{SUMMARY}

Chlamydia gallinacea, a new chlamydial agent, has been reported in four European countries as well as Argentina and China. Experimentally infected chickens with $C$. gallinacea in previous study showed no clinical signs but had significantly reduced gains in body weight $(6 \cdot 5-11 \cdot 4 \%)$. Slaughterhouse workers exposed to infected chickens have developed atypical pneumonia, indicating C. gallinacea is likely a zoonotic agent. In this study, FRET-PCR confirmed that C. gallinacea was present in 12.4\% (66/531) of oral-pharyngeal samples from Alabama backyard poultry. Phylogenetic comparisons based on ompA variable domain showed that 16 sequenced samples represented 14 biotypes. We report for the first time the presence of $C$. gallinacea in North America, and this warrants further research on the organism's pathogenicity, hosts, transmission, and zoonotic potential.

Key words: Chlamydia gallinacea, backyard poultry, FRET-PCR.

Chlamydia spp. are obligate intracellular bacterial pathogens that cause a number of important diseases in animals and humans. Novel members of the group have been discovered in the last few years [1-5] including Chlamydia gallinacea, which was found primarily in chickens, but was also shown to infect cattle [6-8]. Experimentally infected chickens exhibit no clinical disease symptoms but grow more slowly and have significantly reduced gains in body weight $(6 \cdot 5-11 \cdot 4 \%)$ [7]. Importantly, French slaughterhouse workers exposed to C. gallinacea-infected chickens developed respiratory clinical symptoms, suggesting that $C$. gallinacea may also infect humans and may represent a zoonotic

\footnotetext{
* Author for correspondence: C. Wang, College of Veterinary Medicine, Auburn University, Auburn, Alabama, USA. (Email: wangche@auburn.edu)
}

threat [6]. The organism appears to be widely distributed and has been reported in four European countries as well as in Argentina and China [1,6-8]. To determine if this emerging agent might also be present in North America, we used PCR to determine if backyard poultry from Alabama is infected with C. gallinacea.

All samples used in this study were collected for the avian influenza and avian paramyxovirus type-1 surveillance program in avian species organized by the US Department of Agriculture (USDA). Sample collections followed the recommendation for collecting swab specimens from poultry for viral diagnostic testing (USDA SOP WI-AV-0020). In total, 1929 oralpharyngeal swabs from apparently healthy poultry were collected into 531 tubes (1-5 swabs from the same bird and same owner/per tube) containing $3 \mathrm{ml}$ brain-heart infusion (BHI) medium and transported 
(a) : 16S rRNA

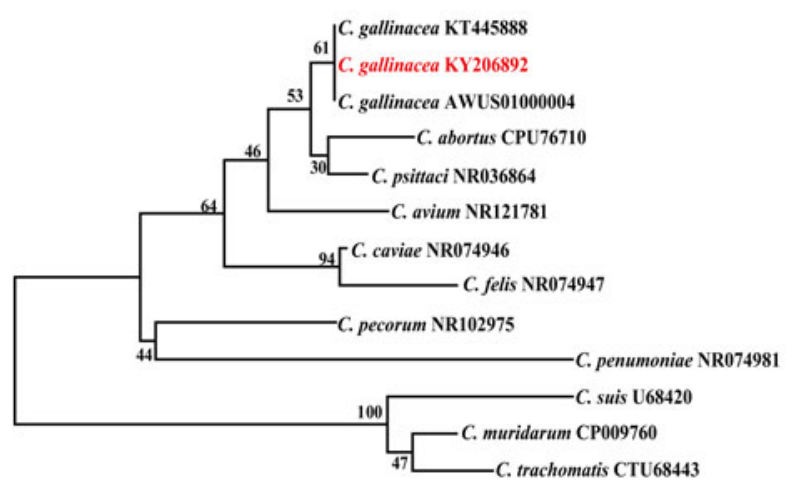

(b) : 23S rRNA

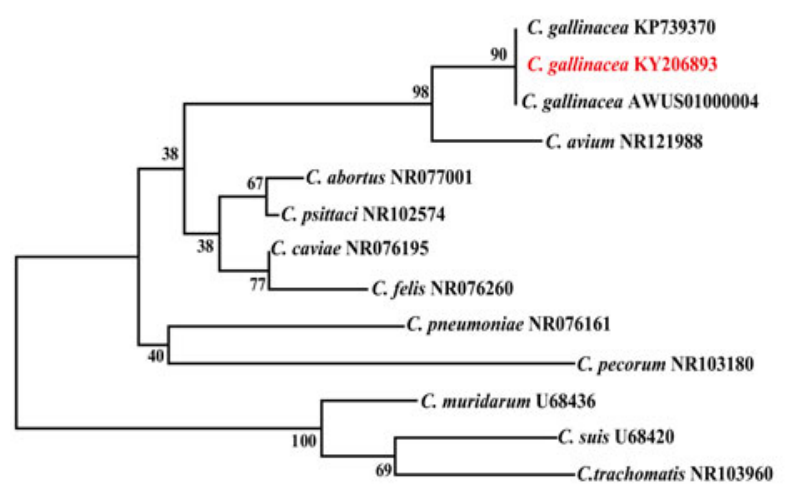

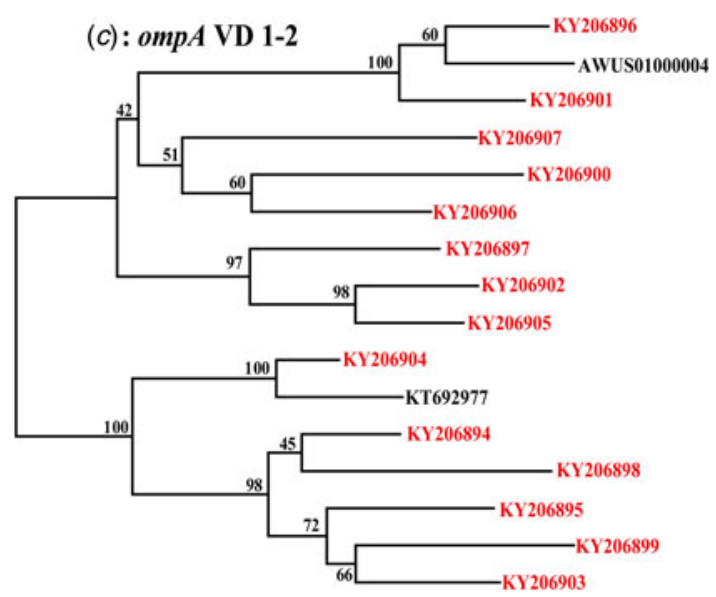

$(d):$ ompA VD 3-4

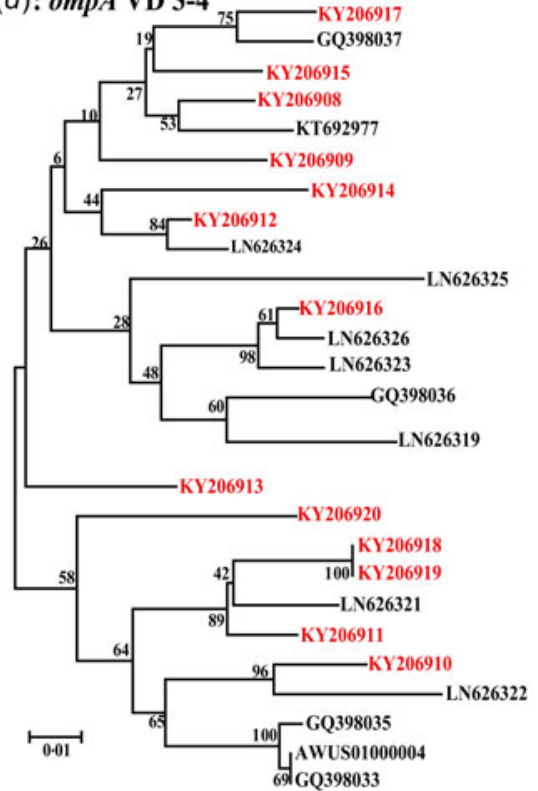

Fig. 1. Phylogenetic comparison of Chlamydia gallinacea from backyard poultry in this study. The 16S rRNA (301 bp) and $23 \mathrm{~S}$ rRNA (329 bp) gene sequences (in red font and accession number) found in the current study are compared with those of other representative Chlamydia spp. (in black font with GenBank accession number) using the Clustalx 1.83 alignment software. In addition, the omp $A$ VD 1-2 (478 bp) and omp $A$ VD 3-4 (311 bp) of C. gallinacea strains identified in in this study are compared with other $C$. gallinacea from poultry in European countries and China. Based on these alignments, phylogenetic trees were constructed by the neighbor-joining method using the Kimura 2-parameter model with MEGA 6.0. Branch lengths are measured in nucleotide substitutions and numbers show branching percentages in bootstrap replicates. Scale bar represents the percent sequence diversity.

on ice to the Alabama State Diagnostic Laboratory. For DNA extraction, BHI for each tube was thawed at room temperature and a $50 \mu 1$ aliquot processed using the MagMax 96 Viral RNA isolation kit (ThermoFisher Scientific, Waltham, MA, USA). The extracted DNA was collected in $50 \mu$ l elution buffer and tested for Chlamydia spp. using a previously described Chlamydia spp. real-time FRET-PCR [7]. This PCR amplifies a 168-bp fragment of the 23S rRNA gene of Chlamydia spp. and can detect all 11 Chlamydia species with a detection sensitivity of a single copy/reaction. High-resolution melting curve analysis enables differentiation of the Chlamydia spp. detected [7].

To further characterize the organisms present, we analyzed high-copy $C$. gallinacea-positive specimens by standard PCRs targeting a variable region of the 16S rRNA (301 bp) of the recognized 11 Chlamydia spp. and a highly variable domain (VD) of the $23 \mathrm{~S}$ rRNA (329 bp) [8,9]. In addition, C. gallinaceaspecific PCRs targeting the omp $A$ VDs $1-2(478 \mathrm{bp})$ and VD 3-4 (311 bp) [8] were used to investigate the 
polymorphism of $C$. gallinacea in the backyard poultry studied (Fig. 1). All PCR products were verified by gel electrophoresis and automated DNA sequencing (Elim Biopharmaceuticals, Inc., Hayward, California, USA).

Altogether $12 \cdot 4 \%(66 / 531)$ of the tubes containing the 1929 oral-pharyngeal samples that we collected from backyard poultry in Alabama were positive in the Chlamydia FRET-PCR. The positives comprised 64 of the 472 tubes containing swabs from chickens $(13.6 \%)$ and two of the six tubes containing swabs from guinea fowl $(33 \cdot 3 \%)$. None of the tubes with swabs from turkeys, geese, ducks, quail, pheasants, peafowl, and pigeons were positive. The 16S rRNA and $23 \mathrm{~S}$ rRNA gene sequences showed that only C. gallinacea was detected, and the strains found in this study were identical to those from Europe and China (Fig. $1 a$ and $b$ ). Sixteen samples positive in the genus-specific Chlamydia-FRET-PCR with over 1000 copies of the $23 \mathrm{~S} \mathrm{rRNA} /$ reaction were further tested with standard PCRs targeting the ompA VD 1-2 and omp A 3-4 domains of Chlamydia spp. [7,8]. Phylogenetic comparisons showed that 16 sequenced samples represented 14 biotypes (Fig. $1 c$ and $d$ ), and the sequences were deposited in GenBank (KY206892, KY206893, KY206894-KY206907, and KY206908KY206920) (Fig. 1 $a-d$ ). The value of phylogenetic comparisons based on partial gene sequences of omp $A$ is limited. Research is in progress to isolate $C$. gallinacea from swabs of backyard poultry, which allows amplification of the whole ompA sequences for complete phylogenetic comparisons.

Avian chlamydiosis is an important infectious disease of domestic poultry as well as companion and wild birds that can be associated with acute, subacute, or chronic respiratory, digestive, or systemic signs. Although $C$. psittaci was considered to be the sole causative agent of avian chlamydiosis, recent studies indicated that prevalence of $C$. gallinacea may be higher than that of C. psittaci $[7,10]$. A survey conducted over 1 year in a French abattoir demonstrated $80.0 \%$ of the Chlamydiaceae-positive samples contained C. gallinacea [6]. Our study in the USA adds to the growing evidence that $C$. gallinacea is a common and widespread chlamydial agent in poultry.

In conclusion, we report for the first time the presence of $C$. gallinacea in North America. The findings from our study should alert the poultry industry in North America to the possibility of a newly emerging pathogen, C. gallinacea, in their flocks. Further studies are indicated to more definitively establish the organism's pathogenicity, hosts, transmission, and zoonotic potential. Such studies will be aided by Chlamydia species-specific peptide ELISAs that have recently become available [11].

\section{ACKNOWLEDGEMENTS}

The authors sincerely thank Professor Patrick Kelly from Ross University School of Veterinary Medicine for the critical reading. This study is supported, in part, by the Alabama Department of Agriculture and Industries for the sample collection and preparation.

\section{DECLARATION OF INTEREST}

The authors declare that no competing financial interests exist.

\section{REFERENCES}

1. Sachse K, et al. Evidence for the existence of two new members of the family Chlamydiaceae and proposal of Chlamydia avium sp. nov. and Chlamydia gallinacea $\mathrm{sp}$. nov. Systemic and Applied Microbiology 2014; 37(2): 79-88.

2. Szymańska-Czerwińska M, Niemczuk K. Avian chlamydiosis zoonotic disease. Vector Borne Zoonotic Diseases 2016; 16(1): 1-3.

3. Laroucau $\mathbf{K}$, et al. Outbreak of psittacosis in a group of women exposed to Chlamydia psittaci-infected chickens. European Surveillance 2015; 20(24): pii: 21155.

4. Vorimore F, et al. Isolation of a new Chlamydia species from the Feral Sacred Ibis (Threskiornis aethiopicus): Chlamydia ibidis. PLoS ONE 2013; 8(9): e74823.

5. Taylor-Brown A, Bachmann NL, Borel N, Polkinghorne A. Culture-independent genomic characterisation of Candidatus Chlamydia sanzinia, a novel uncultivated bacterium infecting snakes. BMC Genomics 2016; 17: 710.

6. Hulin V, et al. Host preference and zoonotic potential of Chlamydia psittaci and $C$. gallinacea in poultry. Pathogens and Diseases 2015; 73(1): 1-11.

7. Guo W, et al. Chlamydia gallinacea, not C. psittaci, is the endemic chlamydial species in chicken (Gallus gallus). Scientific Reports 2016; 6: 19638.

8. Li $\mathbf{J}$, et al. Chlamydia pecorum is the endemic intestinal species in cattle while C. gallinacea, C. psittaci and $C$. pneumoniae associate with sporadic systemic infection. Veterinary Microbiology 2016; 193: 93-99.

9. Frutos MC, et al. Genetic diversity of Chlamydia among captive birds from central Argentina. Avian Pathology 2015; 44(1): 50-56.

10. Sachse K, Laroucau K, Vanrompay D. Avian chlamydiosis. Current Clinical Microbiology Reports 2015; 2: 10-21.

11. Rahman KS, et al. Defining species-specific immunodominant B cell epitopes for molecular serology of Chlamydia species. Clinical and Vaccine Immunology 2015; 22: 539-552. 